\section{$\begin{array}{llllll}\text { I } & \mathbf{N} & \mathbf{F} & \mathbf{S} & \mathbf{Y} & \mathbf{S}\end{array}$} R E S E A R C H $\begin{array}{llllll}\mathbf{R} & \mathrm{E} & \mathrm{P} & \mathrm{O} & \mathrm{R} & \mathrm{T}\end{array}$

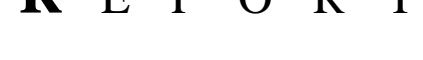
Institut für Informationssysteme AB Wissensbasierte Systeme Technische Universität Wien Favoritenstraße 9-11 A-1040 Wien, Austria Tel: $\quad+43-1-58801-18405$ Fax: +43-1-58801-18493 sek@kr.tuwien.ac.at www.kr.tuwien.ac.at

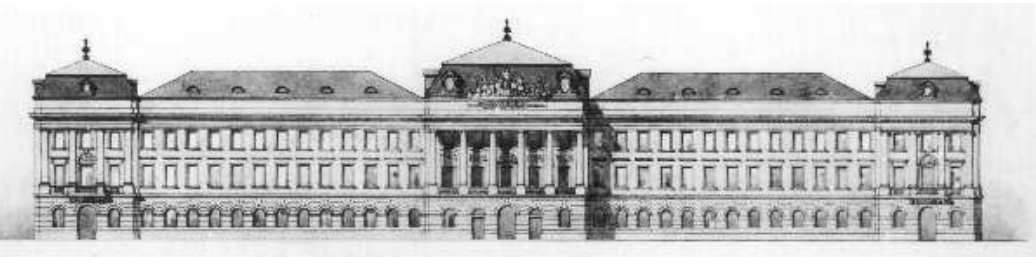

INSTITUT FÜR INFORMATIONSSYSTEME

ARbeitsbereich Wissensbasierte Systeme

\section{PROBABILISTIC DESCRIPTION LOGIC PROGRAMS}

THOMAS LUKASIEWICZ

INFSYS RESEARCH REPORT 1843-06-04

JUNE 2006

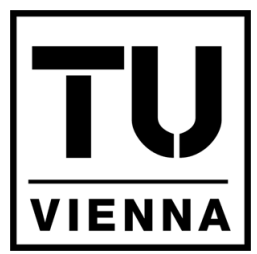



INFSYS RESEARCH REPORT

INFSYS RESEARCH REPORT 1843-06-04, JUNE 2006

\title{
PROBABILISTIC DESCRIPTION LOGIC PROGRAMS
}

\author{
JUNE 10, 2006
}

\author{
Thomas Lukasiewicz ${ }^{1}$
}

\begin{abstract}
Towards sophisticated representation and reasoning techniques that allow for probabilistic uncertainty in the Rules, Logic, and Proof layers of the Semantic Web, we present probabilistic description logic programs (or pdl-programs), which are a combination of description logic programs (or dl-programs) under the answer set semantics and the well-founded semantics with Poole's independent choice logic. We show that query processing in such pdl-programs can be reduced to computing all answer sets of dl-programs and solving linear optimization problems, and to computing the well-founded model of dl-programs, respectively. Moreover, we show that the answer set semantics of pdl-programs is a refinement of the well-founded semantics of pdl-programs. Furthermore, we also present an algorithm for query processing in the special case of stratified pdl-programs, which is based on a reduction to computing the canonical model of stratified dl-programs.
\end{abstract}

\footnotetext{
${ }^{1}$ Dipartimento di Informatica e Sistemistica, Università di Roma "La Sapienza", Via Salaria 113, I-00198 Rome, Italy; e-mail: lukasiewicz@ dis.uniroma1.it. Institut für Informationssysteme, Technische Universität Wien, Favoritenstraße 9-11, A-1040 Vienna, Austria; e-mail: lukasiewicz@kr.tuwien.ac.at.

Acknowledgements: This work has been supported by a Heisenberg Professorship of the German Research Foundation (DFG). I am thankful to the reviewers of the ECSQARU-2005 and URSW-2005 abstracts of this paper for their constructive comments, which helped to improve this work.
}

Copyright (C) 2006 by the authors 


\section{Contents}

1 Introduction 1

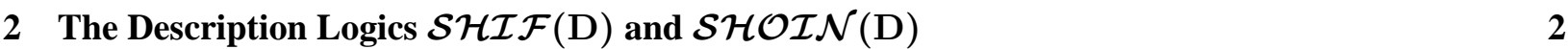

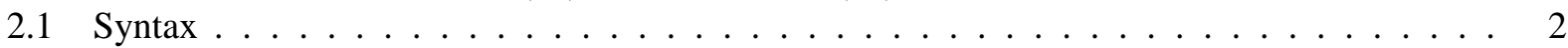

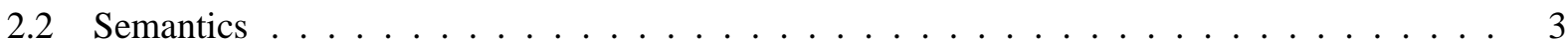

3 Description Logic Programs 4

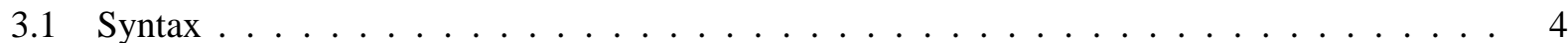

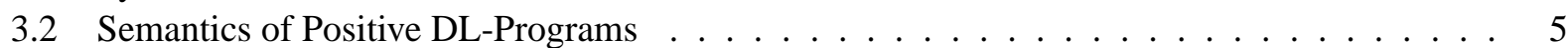

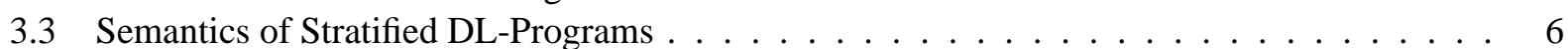

3.4 Answer Set Semantics of DL-Programs . . . . . . . . . . . . . . . . . . . 6

3.5 Well-Founded Semantics of DL-Programs . . . . . . . . . . . . . . . . . . 7

4 Probabilistic Description Logic Programs $\quad 8$

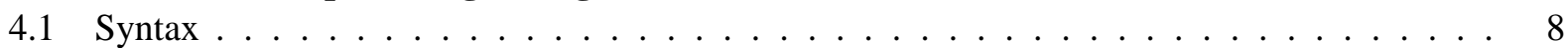

4.2 Semantics of Stratified PDL-Programs _ . . . . . . . . . . . . . . . . . . . 9

4.3 Answer Set Semantics of PDL-Programs . . . . . . . . . . . . . . . . . . . . . . . 10

4.4 Well-Founded Semantics of PDL-Programs . . . . . . . . . . . . . . . . . . 11

5 Query Processing in Stratified PDL-Programs 12

5.1 Fixpoint Iteration in Positive DL-Programs . . . . . . . . . . . . . . . . . . . . 13

5.2 Fixpoint Iteration in Stratified DL-Programs . . . . . . . . . . . . . . . . . . . 13

5.3 Query Processing in Stratified PDL-Programs . . . . . . . . . . . . . . . . . . . 13

6 Related Work 14

6.1 Description Logic Programs . . . . . . . . . . . . . . . . . . . . . 15

6.2 Uncertainty Reasoning for the Semantic Web . . . . . . . . . . . . . . . . . . 15

7 Conclusion $\quad 16$ 


\section{Introduction}

The Semantic Web [5, 14] aims at an extension of the current World Wide Web by standards and technologies that help machines to understand the information on the Web so that they can support richer discovery, data integration, navigation, and automation of tasks. The main ideas behind it are to add a machine-readable meaning to Web pages, to use ontologies for a precise definition of shared terms in Web resources, to use KR technology for automated reasoning from Web resources, and to apply cooperative agent technology for processing the information of the Web.

The Semantic Web consists of several hierarchical layers, where the Ontology layer, in form of the OWL Web Ontology Language [46, 23] (recommended by the W3C), is currently the highest layer of sufficient maturity. OWL consists of three increasingly expressive sublanguages, namely $O W L$ Lite, $O W L D L$, and OWL Full. OWL Lite and OWL DL are essentially very expressive description logics with an RDF syntax [23]. As shown in [21], ontology entailment in OWL Lite (resp., OWL DL) reduces to knowledge base

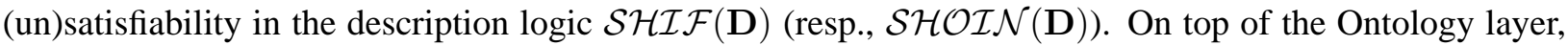
the Rules, Logic, and Proof layers of the Semantic Web will be developed next, which should offer sophisticated representation and reasoning capabilities. As a first effort in this direction, RuleML (Rule Markup Language) [6] is an XML-based markup language for rules and rule-based systems, whereas the OWL Rules Language [22] is a first proposal for extending OWL by Horn clause rules.

A key requirement of the layered architecture of the Semantic Web is to integrate the Rules and the Ontology layer. In particular, it is crucial to allow for building rules on top of ontologies, that is, for rulebased systems that use vocabulary from ontology knowledge bases. Another type of combination is to build ontologies on top of rules, which means that ontological definitions are supplemented by rules or imported from rules. Towards this goal, the works $[12,13]$ have proposed description logic programs (or simply dlprograms), which are of the form $K B=(L, P)$, where $L$ is a knowledge base in a description logic and $P$ is a finite set of description logic rules (or simply dl-rules). Such dl-rules are similar to usual rules in logic programs with negation as failure, but may also contain queries to $L$ in their bodies, which are given by special atoms (on which possibly default negation may apply). Another important feature of dl-rules is that queries to $L$ also allow for specifying an input from $P$, and thus for a flow of information from $P$ to $L$, besides the flow of information from $L$ to $P$, given by any query to $L$. Hence, description logic programs allow for building rules on top of ontologies, but also (to some extent) building ontologies on top of rules. In this way, additional knowledge (gained in the program) can be supplied to $L$ before querying. The semantics of dl-programs was defined in [12] and [13] as an extension of the answer set semantics by Gelfond and Lifschitz [17] and the well-founded semantics by Van Gelder, Ross, and Schlipf [45], respectively, which are the two most widely used semantics for nonmonotonic logic programs. The description logic knowledge

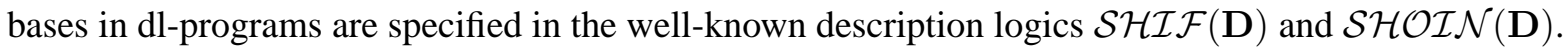

In this paper, we continue this line of research. Towards sophisticated representation and reasoning techniques that also allow for modeling probabilistic uncertainty in the Rules, Logic, and Proof layers of the Semantic Web, we present probabilistic description logic programs (or simply pdl-programs), which generalize dl-programs under the answer set and the well-founded semantics by probabilistic uncertainty. This probabilistic generalization of dl-programs is developed as a combination of dl-programs with Poole's independent choice logic (ICL) [35].

It is important to point out that Poole's ICL is a powerful representation and reasoning formalism for single- and also multi-agent systems, which combines logic and probability, and which can represent a number of important uncertainty formalisms, in particular, influence diagrams, Bayesian networks, Markov decision processes, and normal form games [35]. Furthermore, Poole's ICL also allows for natural notions 
of causes and explanations as in Pearl's structural causal models [15].

To my knowledge, this is the first work that combines description logic programs with probabilistic uncertainty. The main contributions are summarized as follows:

- We present probabilistic description logic programs (or pdl-programs), which are a probabilistic generalization of dl-programs $[12,13]$. They are a combination of dl-programs with Poole's independent choice logic (ICL) [35]; they properly generalize ICL programs (with finite Herbrand bases) by description logics.

- We define a probabilistic answer set semantics of pdl-programs, which is a generalization of the (strong) answer set semantics of dl-programs in [12]. We show that query processing in pdl-programs under this semantics is reducible to computing all answer sets of dl-programs and solving linear optimization problems.

- We define a probabilistic well-founded semantics of pdl-programs, which is a generalization of the well-founded semantics of dl-programs in [13]. We then show that query processing in pdl-programs under the well-founded semantics can be reduced to computing the well-founded semantics of dlprograms.

- We show that, like for the case of dl-programs, the answer set semantics of pdl-programs is a refinement of the well-founded semantics of pdl-programs. That is, whenever an answer to a query under the well-founded semantics is defined, it coincides with the answer to the query under the answer set semantics.

- We also present an algorithm for query processing in the special case of stratified pdl-programs. It is based on a reduction to computing the canonical model of stratified dl-programs, which can be done by a finite sequence of finite fixpoint iterations. This shows especially that query processing in stratified pdl-programs is conceptually easier than query processing in general pdl-programs.

The rest of this paper is organized as follows. Section 2 recalls the description logics $\mathcal{S H \mathcal { H }}(\mathbf{D})$ and $\mathcal{S H O I N}(\mathbf{D})$. In Section 3, we recall dl-programs under the stratified, the answer set, and the well-founded semantics. In Section 4, we introduce their probabilistic generalization to pdl-programs. Section 5 focuses on query processing in stratified pdl-programs. In Sections 6 and 7, we discuss related work, summarize the main results, and give an outlook on future research.

\section{The Description Logics $\mathcal{S H} \mathcal{H} \mathcal{F}(\mathrm{D})$ and $\mathcal{S H O} \mathcal{I} \mathcal{N}(\mathrm{D})$}

In this section, we recall the description logics $\mathcal{S H \mathcal { I F }}(\mathbf{D})$ and $\mathcal{S H O} \mathcal{H} \mathcal{N}(\mathbf{D})$.

\subsection{Syntax}

We first describe the syntax of $\mathcal{S H O I N}(\mathbf{D})$. We assume a set of elementary datatypes and a set of data values. A datatype is either an elementary datatype or a set of data values (called datatype oneOf). A datatype theory $\mathbf{D}=\left(\Delta^{\mathbf{D}},{ }^{\mathbf{D}}\right)$ consists of a datatype (or concrete) domain $\Delta^{\mathbf{D}}$ and a mapping ${ }^{\mathbf{D}}$ that associates with every elementary datatype a subset of $\Delta^{\mathbf{D}}$ and with every data value an element of $\Delta^{\mathbf{D}}$. The mapping ${ }^{\mathbf{D}}$ is extended to all datatypes by $\left\{v_{1}, \ldots\right\}^{\mathbf{D}}=\left\{v_{1}^{\mathbf{D}}, \ldots\right\}$. Let $\mathbf{A}, \mathbf{R}_{A}, \mathbf{R}_{D}$, and $\mathbf{I}$ be nonempty finite and pairwise 
disjoint sets of atomic concepts, abstract roles, datatype (or concrete) roles, and individuals, respectively. We denote by $\mathbf{R}_{A}^{-}$the set of inverses $R^{-}$of all $R \in \mathbf{R}_{A}$.

A role is an element of $\mathbf{R}_{A} \cup \mathbf{R}_{A}^{-} \cup \mathbf{R}_{D}$. Concepts are inductively defined as follows. Every $C \in \mathbf{A}$ is a concept, and if $o_{1}, \ldots, o_{n} \in \mathbf{I}$, then $\left\{o_{1}, \ldots, o_{n}\right\}$ is a concept (called oneOf). If $C, C_{1}$, and $C_{2}$ are concepts and if $R \in \mathbf{R}_{A} \cup \mathbf{R}_{A}^{-}$, then also $\left(C_{1} \sqcap C_{2}\right),\left(C_{1} \sqcup C_{2}\right)$, and $\neg C$ are concepts (called conjunction, disjunction, and negation, respectively), as well as $\exists R . C, \forall R . C, \geq n R$, and $\leq n R$ (called exists, value, atleast, and atmost restriction, respectively) for an integer $n \geq 0$. If $D$ is a datatype and $U \in \mathbf{R}_{D}$, then $\exists U . D, \forall U . D$, $\geq n U$, and $\leq n U$ are concepts (called datatype exists, value, atleast, and atmost restriction, respectively) for an integer $n \geq 0$. We write $\top$ and $\perp$ to abbreviate the concepts $C \sqcup \neg C$ and $C \sqcap \neg C$, respectively, and we eliminate parentheses as usual.

An axiom is an expression of one of the following forms: (1) $C \sqsubseteq D$ (called concept inclusion axiom), where $C$ and $D$ are concepts; (2) $R \sqsubseteq S$ (called role inclusion axiom), where either $R, S \in \mathbf{R}_{A}$ or $R, S \in \mathbf{R}_{D}$; (3) Trans $(R)$ (called transitivity axiom), where $R \in \mathbf{R}_{A}$; (4) $C(a)$ (called concept membership axiom), where $C$ is a concept and $a \in \mathbf{I}$; (5) $R(a, b)$ (resp., $U(a, v)$ ) (called role membership axiom), where $R \in \mathbf{R}_{A}$ (resp., $U \in \mathbf{R}_{D}$ ) and $a, b \in \mathbf{I}$ (resp., $a \in \mathbf{I}$ and $v$ is a data value); and (6) $a=b$ (resp., $a \neq b$ ) (called equality (resp., inequality) axiom), where $a, b \in \mathbf{I}$. A knowledge base $L$ is a finite set of axioms. For decidability, number restrictions in $L$ are restricted to simple abstract roles $R \in \mathbf{R}_{A}$ [24].

The syntax of $\mathcal{S H \mathcal { F }}(\mathbf{D})$ is as the above syntax of $\mathcal{S H O I N}(\mathbf{D})$, but without the oneOf constructor and with the atleast and atmost constructors limited to 0 and 1.

Example 2.1 An online store (such as amazon.com) may use a description logic knowledge base to classify and characterize its products. For example, suppose that (1) textbooks are books, (2) personal computers and cameras are electronic products, (3) books and electronic products are products, (4) every product has at least one related product, (5) only products are related to each other, (6) $t b_{-} a i$ and $t b_{-} l p$ are textbooks, which are related to each other, (7) $p c_{-} i b m$ and $p c_{-} h p$ are personal computers, which are related to each other, and (8) $i b m$ and $h p$ are providers for $p c_{-} i b m$ and $p c_{-} h p$, respectively. This knowledge is expressed by the following description logic knowledge base $L_{1}$ in $\mathcal{S H \mathcal { H }} \mathcal{F}(\mathbf{D})$ :

(1) Textbook $\sqsubseteq$ Book; (2) PC $\sqcup$ Camera $\sqsubseteq$ Electronics;

(3) Book $\sqcup$ Electronics $\sqsubseteq$ Product; (4) Product $\sqsubseteq \geq 1$ related;

(5) $\geq 1$ related $\sqcup \geq 1$ related $^{-} \sqsubseteq$ Product;

(6) Textbook(tb_ai); Textbook $\left(t b_{-} l p\right)$; related $\left(t b_{-} a i, t b \_l p\right)$;

(7) $P C\left(p c_{\_} i b m\right) ; P C\left(p c \_h p\right)$; related $\left(p c_{\_} i b m, p c \_h p\right)$;

(8) provides $\left(i b m, p c \_i b m\right)$; provides $\left(h p, p c \_h p\right)$.

\subsection{Semantics}

An interpretation $\mathcal{I}=\left(\Delta^{\mathcal{I}},{ }^{\mathcal{I}}\right)$ with respect to a datatype theory $\mathbf{D}=\left(\Delta^{\mathbf{D}}, .^{\mathbf{D}}\right)$ consists of a nonempty (abstract) domain $\Delta^{\mathcal{I}}$ disjoint from $\Delta^{\mathbf{D}}$, and a mapping ${ }^{\mathcal{I}}$ that assigns to each atomic concept $C \in \mathbf{A}$ a subset of $\Delta^{\mathcal{I}}$, to each individual $o \in \mathbf{I}$ an element of $\Delta^{\mathcal{I}}$, to each abstract role $R \in \mathbf{R}_{A}$ a subset of $\Delta^{\mathcal{I}} \times \Delta^{\mathcal{I}}$, and to each datatype role $U \in \mathbf{R}_{D}$ a subset of $\Delta^{\mathcal{I}} \times \Delta^{\mathbf{D}}$ ). The mapping ${ }^{\mathcal{I}}$ is extended to all concepts and roles as usual (where $\# S$ denotes the cardinality of a set $S$ ):

- $\left\{o_{1}, \ldots, o_{n}\right\}^{\mathcal{I}}=\left\{o_{1}^{\mathcal{I}}, \ldots, o_{n}^{\mathcal{I}}\right\}$ 
- $(C \sqcap D)^{\mathcal{I}}=C^{\mathcal{I}} \cap D^{\mathcal{I}},(C \sqcup D)^{\mathcal{I}}=C^{\mathcal{I}} \cup D^{\mathcal{I}}$, and $(\neg C)^{\mathcal{I}}=\Delta^{\mathcal{I}} \backslash C^{\mathcal{I}}$;

- $(\exists R . C)^{\mathcal{I}}=\left\{x \in \Delta^{\mathcal{I}} \mid \exists y:(x, y) \in R^{\mathcal{I}} \wedge y \in C^{\mathcal{I}}\right\}$;

- $(\forall R . C)^{\mathcal{I}}=\left\{x \in \Delta^{\mathcal{I}} \mid \forall y:(x, y) \in R^{\mathcal{I}} \rightarrow y \in C^{\mathcal{I}}\right\}$;

- $(\geq n R)^{\mathcal{I}}=\left\{x \in \Delta^{\mathcal{I}} \mid \#\left(\left\{y \mid(x, y) \in R^{\mathcal{I}}\right\}\right) \geq n\right\}$;

- $(\leq n R)^{\mathcal{I}}=\left\{x \in \Delta^{\mathcal{I}} \mid \#\left(\left\{y \mid(x, y) \in R^{\mathcal{I}}\right\}\right) \leq n\right\}$;

- $(\exists U . D)^{\mathcal{I}}=\left\{x \in \Delta^{\mathcal{I}} \mid \exists y:(x, y) \in U^{\mathcal{I}} \wedge y \in D^{\mathbf{D}}\right\}$;

- $(\forall U . D)^{\mathcal{I}}=\left\{x \in \Delta^{\mathcal{I}} \mid \forall y:(x, y) \in U^{\mathcal{I}} \rightarrow y \in D^{\mathbf{D}}\right\}$;

- $(\geq n U)^{\mathcal{I}}=\left\{x \in \Delta^{\mathcal{I}} \mid \#\left(\left\{y \mid(x, y) \in U^{\mathcal{I}}\right\}\right) \geq n\right\}$;

- $(\leq n U)^{\mathcal{I}}=\left\{x \in \Delta^{\mathcal{I}} \mid \#\left(\left\{y \mid(x, y) \in U^{\mathcal{I}}\right\}\right) \leq n\right\}$.

The satisfaction of a description logic axiom $F$ in the interpretation $\mathcal{I}=\left(\Delta^{\mathcal{I}},{ }^{\mathcal{I}}\right)$ with respect to $\mathbf{D}=$ $\left(\Delta^{\mathbf{D}},{ }^{\mathbf{D}}\right)$, denoted $\mathcal{I} \models F$, is defined as follows: (1) $\mathcal{I} \models C \sqsubseteq D$ iff $C^{\mathcal{I}} \subseteq D^{\mathcal{I}}$; (2) $\mathcal{I} \models R \sqsubseteq S$ iff $R^{\mathcal{I}} \subseteq S^{\mathcal{I}}$; (3) $\mathcal{I} \models \operatorname{Trans}(R)$ iff $R^{\mathcal{I}}$ is transitive; (4) $\mathcal{I} \models C(a)$ iff $a^{\overline{\mathcal{I}}} \in C^{\mathcal{I}}$; (5) $\overline{\mathcal{I}} \models R(a, b)$ iff $\left(\bar{a}^{\mathcal{I}}, b^{\mathcal{I}}\right) \in R^{\overline{\mathcal{I}}}$; (6) $\mathcal{I} \models U(a, v)$ iff $\left(a^{\mathcal{I}}, v^{\mathbf{D}}\right) \in U^{\mathcal{I}}$; (7) $\mathcal{I}=a=b$ iff $a^{\mathcal{I}}=b^{\mathcal{I}}$; and (8) $\mathcal{I}=a \neq b$ iff $a^{\mathcal{I}} \neq b^{\mathcal{I}}$. The interpretation $\mathcal{I}$ satisfies the axiom $F$, or $\mathcal{I}$ is a model of $F$, iff $\mathcal{I} \models F$. The interpretation $\mathcal{I}$ satisfies a knowledge base $L$, or $\mathcal{I}$ is a model of $L$, denoted $\mathcal{I} \models L$, iff $\mathcal{I} \models F$ for all $F \in L$. We say that $L$ is satisfiable (resp., unsatisfiable) iff $L$ has a (resp., no) model. An axiom $F$ is a logical consequence of $L$, denoted $L \models F$, iff every model of $L$ satisfies $F$. A negated axiom $\neg F$ is a logical consequence of $L$, denoted $L \models \neg F$, iff every model of $L$ does not satisfy $F$.

\section{Description Logic Programs}

In this section, we recall description logic programs (or dl-programs) [12,13], which are a combination of description logics and normal programs. They consist of a knowledge base $L$ in a description logic and a finite set of description logic rules $P$. Such rules are similar to usual rules in logic programs with negation as failure, but may also contain queries to $L$, possibly default negated. We describe the canonical semantics of positive and stratified dl-programs, as well as the answer set semantics and the well-founded semantics of general dl-programs.

\subsection{Syntax}

We now define the syntax of dl-programs. We first define the syntax of ordinary normal rules and of ordinary normal and positive programs.

We assume a function-free first-order vocabulary $\Phi$ with nonempty finite sets of constant and predicate symbols, and a set of variables $\mathcal{X}$. A term is a constant symbol from $\Phi$ or a variable from $\mathcal{X}$. If $p$ is a predicate symbol of arity $k \geq 0$ from $\Phi$ and $t_{1}, \ldots, t_{k}$ are terms, then $p\left(t_{1}, \ldots, t_{k}\right)$ is an atom. A negationas-failure literal is an atom $a$ or a default-negated atom not $a$. A normal rule $r$ is of the form

$$
a \leftarrow b_{1}, \ldots, b_{k}, \text { not } b_{k+1}, \ldots, \text { not } b_{m}, m \geq k \geq 0,
$$

where $a, b_{1}, \ldots, b_{m}$ are atoms. We refer to $a$ as the head of $r$, denoted $H(r)$, while the conjunction $b_{1}, \ldots, b_{k}$, not $b_{k+1}, \ldots$, not $b_{m}$ is called the body of $r$; its positive (resp., negative) part is $b_{1}, \ldots, b_{k}$ (resp., not $b_{k+1}, \ldots$, not $b_{m}$ ). We define $B(r)=B^{+}(r) \cup B^{-}(r)$, where $B^{+}(r)=\left\{b_{1}, \ldots, b_{k}\right\}$ and 
$B^{-}(r)=\left\{b_{k+1}, \ldots, b_{m}\right\}$. A normal program $P$ is a finite set of normal rules. We say that $P$ is positive iff no rule in $P$ contains default-negated atoms.

We next define the syntax of dl-programs. Informally, they consist of a description logic knowledge base $L$ and a generalized normal program $P$, which may contain queries to $L$. In such a query, it is asked whether a certain description logic axiom or its negation logically follows from $L$ or not. Formally, a $d l$ query $Q(\mathbf{t})$ is either

(a) a concept inclusion axiom $F$ or its negation $\neg F$; or

(b) of the forms $C(t)$ or $\neg C(t)$, where $C$ is a concept and $t$ is a term; or

(c) of the forms $R\left(t_{1}, t_{2}\right)$ or $\neg R\left(t_{1}, t_{2}\right)$, where $R$ is a role and $t_{1}, t_{2}$ are terms.

A dl-atom has the form $D L\left[S_{1} o p_{1} p_{1}, \ldots, S_{m} o p_{m} p_{m} ; Q\right](\mathbf{t})$, where each $S_{i}$ is a concept resp. role, $o p_{i} \in$ $\{\uplus, \uplus\}$ resp. $o p_{i}=\uplus, p_{i}$ is a unary resp. binary predicate symbol, $Q(\mathbf{t})$ is a dl-query, and $m \geq 0$. We call $p_{1}, \ldots, p_{m}$ its input predicate symbols. Intuitively, $o p_{i}=\uplus$ (resp., $o p_{i}=\uplus$ ) increases $S_{i}$ (resp., $\neg S_{i}$ ) by the extension of $p_{i}$. A dl-rule $r$ is of the form (1), where any $b \in B(r)$ is either an ordinary atom or a dl-atom. A description logic program (or dl-program) $K B=(L, P)$ consists of a description logic knowledge base $L$ and a finite set of dl-rules $P$. Ground terms, atoms, literals, etc., are defined as usual. The Herbrand base of $P$, denoted $H B_{P}$, is the set of all ground atoms with standard predicate symbols that occur in $P$ and constant symbols in $\Phi$. We denote by $\operatorname{ground}(P)$ the set of all ground instances of dl-rules in $P$ relative to $H B_{P}$.

Example 3.1 Consider the dl-program $K B_{1}=\left(L_{1}, P_{1}\right)$, where $L_{1}$ is the description logic knowledge base from Example 2.1, and $P_{1}$ is the following set of dl-rules:

(1) $p c\left(p c_{-} 1\right) ; \quad p c\left(p c_{-} 2\right) ; \quad p c\left(p c_{-} 3\right)$;

(2) brand_new $\left(p c_{-} 1\right)$; brand_new $\left(p c_{-} 2\right)$;

(3) vendor $\left(\right.$ dell, $\left.p c_{-} 1\right)$; vendor $\left(\right.$ dell, $\left.p c_{-} 2\right)$; vendor $\left(\right.$ dell, $\left.p c_{-} 3\right)$;

(4) $\operatorname{avoid}(X) \leftarrow D L[$ Camera $](X)$, not offer $(X)$;

(5) $\quad$ offer $(X) \leftarrow D L[P C \uplus p c ;$ Electronics $](X)$, not brand_new $(X)$;

(6) $\operatorname{provider}(V) \leftarrow \operatorname{vendor}(V, X), D L[P C \uplus p c ; \operatorname{Product}](X)$;

(7) $\operatorname{provider}(V) \leftarrow D L[$ provides $](V, X), D L[P C \uplus p c ;$ Product $](X)$;

(8) $\operatorname{similar}(X, Y) \leftarrow D L[$ related $](X, Y)$;

(9) $\operatorname{similar}(X, Z) \leftarrow \operatorname{similar}(X, Y)$, $\operatorname{similar}(Y, Z)$.

The above dl-rules express that (1) $p c_{-} 1, p c_{-} 2$, and $p c_{-} 3$ are additional personal computers, (2) $p c_{-} 1$ and $p c_{-} 2$ are brand new, (3) dell is the vendor of $p c_{-} 1, p c_{-} 2$, and $p c_{-} 3$, (4) a customer avoids all cameras that are not on offer, (5) all electronic products that are not brand new are on offer, (6) every vendor of a product is a provider, (7) every entity providing a product is a provider, (8) all related products are similar, and (9) the binary similarity relation on products is transitively closed.

\subsection{Semantics of Positive DL-Programs}

We now define positive dl-programs and their canonical semantics. We first define interpretations and the satisfaction of dl-programs in interpretations.

In the sequel, let $K B=(L, P)$ be a dl-program. An interpretation $I$ relative to $P$ is any $I \subseteq H B_{P}$. We say that $I$ is a model of $a \in H B_{P}$, denoted $I \models a$, iff $a \in I$. We say $I$ is a model of $a \in H B_{P}$ under $L$, 
denoted $I \models_{L} a$, iff $I \models a$. We say $I$ is a model of a ground dl-atom $a=D L\left[S_{1} o p_{1} p_{1}, \ldots, S_{m} o p_{m} p_{m} ; Q\right](\mathbf{c})$ under $L$, denoted $I \models_{L} a$, iff $L \cup \bigcup_{i=1}^{m} A_{i}(I) \models Q(\mathbf{c})$, where $A_{i}(I)=\left\{S_{i}(\mathbf{e}) \mid p_{i}(\mathbf{e}) \in I\right\}$, for $o p_{i}=\uplus$; and $A_{i}(I)=\left\{\neg S_{i}(\mathbf{e}) \mid p_{i}(\mathbf{e}) \in I\right\}$, for $o p_{i}=\forall$. A ground dl-atom $a$ is monotonic relative to $K B=(L, P)$ iff $I \subseteq I^{\prime} \subseteq H B_{P}$ implies that if $I \models_{L} a$ then $I^{\prime} \models_{L} a$. In this paper, we consider only monotonic ground dlatoms, but observe that one can also define dl-atoms that are not monotonic; see [12]. We say that $I$ is a model of a ground dl-rule $r$ under $L$, denoted $I \models_{L} r$, iff $I \models_{L} H(r)$ whenever $I \models_{L} B(r)$, that is, $I \models_{L} a$ for all $a \in B^{+}(r)$ and $I \not \not_{L} a$ for all $a \in B^{-}(r)$. We say $I$ is a model of a dl-program $K B=(L, P)$, denoted $I \models K B$, iff $I \models_{L} r$ for every $r \in \operatorname{ground}(P)$. We say $K B$ is satisfiable (resp., unsatisfiable) iff it has some (resp., no) model.

We say $K B=(L, P)$ is positive iff no dl-rule in $P$ contains default-negated atoms. Like ordinary positive programs, every positive dl-program $K B$ is satisfiable and has a unique least model, denoted $M_{K B}$, that naturally characterizes its semantics.

\subsection{Semantics of Stratified DL-Programs}

We next define stratified dl-programs and their canonical semantics. They are intuitively composed of hierarchic layers of positive dl-programs linked via default negation. Like ordinary stratified normal programs, they are always satisfiable and can be assigned a canonical minimal model via a number of iterative least models.

For any dl-program $K B=(L, P)$, we denote by $D L_{P}$ the set of all ground dl-atoms that occur in ground $(P)$. An input atom of $a \in D L_{P}$ is a ground atom with an input predicate of $a$ and constant symbols in $\Phi$. A (local) stratification of $K B=(L, P)$ is a mapping $\lambda: H B_{P} \cup D L_{P} \rightarrow\{0,1, \ldots, k\}$ such that

(i) $\lambda(H(r)) \geq \lambda\left(b^{\prime}\right)\left(\right.$ resp., $\lambda(H(r))>\lambda\left(b^{\prime}\right)$ ) for every $r \in \operatorname{ground}(P)$ and $b^{\prime} \in B^{+}(r)$ (resp., $\left.b^{\prime} \in B^{-}(r)\right)$, and

(ii) $\lambda(a) \geq \lambda(b)$ for each input atom $b$ of each $a \in D L_{P}$,

where $k \geq 0$ is the length of $\lambda$. For $i \in\{0, \ldots, k\}$, let $K B_{i}=\left(L, P_{i}\right)=(L,\{r \in \operatorname{ground}(P) \mid \lambda(H(r))=$ $i$ \}), and let $H B_{P_{i}}$ (resp., $H B_{P_{i}}^{\star}$ ) be the set of all $b \in H B_{P}$ such that $\lambda(b)=i$ (resp., $\left.\lambda(b) \leq i\right)$. A dl-program $K B=(L, P)$ is (locally) stratified iff it has a stratification $\lambda$ of some length $k \geq 0$. We define its iterative least models $M_{i} \subseteq H B_{P}$ with $i \in\{0, \ldots, k\}$ as follows:

(i) $M_{0}$ is the least model of $K B_{0}$;

(ii) if $i>0$, then $M_{i}$ is the least model of $K B_{i}$ such that $M_{i}\left|H B_{P_{i-1}}^{\star}=M_{i-1}\right| H B_{P_{i-1}}^{\star}$.

The canonical model of the stratified dl-program $K B$, denoted $M_{K B}$, is then defined as $M_{k}$. Observe that $M_{K B}$ is well-defined, since it does not depend on a particular $\lambda$. Furthermore, $M_{K B}$ is in fact a minimal model of $K B$.

\subsection{Answer Set Semantics of DL-Programs}

The answer set semantics of general dl-programs is defined by a reduction to the least model semantics of positive dl-programs as follows. We use a transformation that removes all default-negated atoms in dl-rules and that generalizes the Gelfond-Lifschitz transformation [17]. More precisely, for dl-programs $K B=(L, P)$, the (strong) dl-transform of $P$ relative to $L$ and an interpretation $I \subseteq H B_{P}$, denoted $s P_{L}^{I}$, is the set of all dl-rules obtained from $\operatorname{ground}(P)$ by (i) deleting every dl-rule $r$ such that $I \models_{L} a$ for some 
$a \in B^{-}(r)$, and (ii) deleting from each remaining dl-rule $r$ the negative body. A (strong) answer set of $K B$ is an interpretation $I \subseteq H B_{P}$ such that $I$ is the unique least model of $\left(L, s P_{L}^{I}\right)$.

The answer set semantics of dl-programs $K B=(L, P)$ without dl-atoms coincides with the ordinary answer set semantics of $P$. Answer sets of a general dl-program $K B$ are also minimal models of $K B$. Positive and locally stratified dl-programs have exactly one answer set, which coincides with their canonical minimal model.

\subsection{Well-Founded Semantics of DL-Programs}

In the sequel, let $K B=(L, P)$ be a dl-program. For literals $l=a$ (resp., $l=\neg a$ ), we use $\neg . l$ to denote $\neg a$ (resp., a), and for sets of literals $S$, we define $\neg . S=\{\neg . l \mid l \in S\}$ and $S^{+}=\{a \in S \mid a$ is an atom $\}$. We define Lit $_{P}=H B_{P} \cup \neg$. $H B_{P}$. A set $S \subseteq$ Lit $_{P}$ is consistent iff $S \cap \neg . S=\emptyset$. A three-valued interpretation relative to $P$ is any consistent $I \subseteq L_{i t}$. We define the well-founded semantics of $K B$ by generalizing its standard definition based on unfounded sets [45].

We first define unfounded sets of dl-programs. Let $I \subseteq L_{i t}$ be consistent. A set $U \subseteq H B_{P}$ is an unfounded set of $K B$ relative to $I$ iff the following holds:

(*) for every $a \in U$ and every $r \in \operatorname{ground}(P)$ with $H(r)=a$, either (i) $\neg b \in I \cup \neg$. $U$ for some ordinary atom $b \in B^{+}(r)$, or (ii) $b \in I$ for some ordinary atom $b \in B^{-}(r)$, or (iii) for some dl-atom $b \in B^{+}(r)$, it holds that $S^{+} \not \models_{L} b$ for every consistent $S \subseteq L i t_{P}$ with $I \cup \neg . U \subseteq S$, or (iv) $I^{+} \models_{L} b$ for some dl-atom $b \in B^{-}(r)$.

For every dl-program $K B=(L, P)$ and consistent $I \subseteq L_{i t}$, the set of unfounded sets of $K B$ relative to $I$ is closed under union, and thus $K B$ has a greatest unfounded set relative to $I$, denoted $U_{K B}(I)$. Intuitively, if $I$ is compatible with $K B$, then all atoms in $U_{K B}(I)$ can be safely switched to false and the resulting interpretation is still compatible with $K B$. We define the operators $T_{K B}$ and $W_{K B}$ on all consistent $I \subseteq L i t_{P}$ as follows:

- $a \in T_{K B}(I)$ iff $a \in H B_{P}$ and some $r \in \operatorname{ground}(P)$ exists such that (a) $H(r)=a$, (b) $I^{+} \models_{L} b$ for all $b \in B^{+}(r)$, (c) $\neg b \in I$ for all ordinary atoms $b \in B^{-}(r)$, and (d) $S^{+} \forall_{L} b$ for each consistent $S \subseteq L_{i t} t_{P}$ with $I \subseteq S$, for all dl-atoms $b \in B^{-}(r)$;

- $W_{K B}(I)=T_{K B}(I) \cup \neg . U_{K B}(I)$.

The operators $T_{K B}, U_{K B}$, and $W_{K B}$ are all monotonic. Thus, in particular, $W_{K B}$ has a least fixpoint, denoted $l f p\left(W_{K B}\right)$. The well-founded semantics of $K B=(L, P)$, denoted $W F S(K B)$, is defined as $l f p\left(W_{K B}\right)$. An atom $a \in H B_{P}$ is well-founded (resp., unfounded) relative to $K B$ iff $a$ (resp., $\neg a$ ) is in $W F S(K B)$. Intuitively, starting with $I=\emptyset$, rules are applied to obtain new positive (resp., negated) facts via $T_{K B}(I)$ (resp., ᄀ. $U_{K B}(I)$ ). This process is repeated until no longer possible.

The well-founded semantics of dl-programs $K B=(L, P)$ without dl-atoms coincides with the ordinary well-founded semantics of $P$. In general, $W F S(K B)$ is a partial model of $K B$. Here, a consistent $I \subseteq L_{i t}$ is a partial model of $K B$ iff it can be extended to a (two-valued) model $I^{\prime} \subseteq H B_{P}$ of $K B$. Like in the ordinary case, the well-founded semantics for positive and locally stratified dl-programs is total and coincides with their canonical minimal model. The well-founded semantics for dl-programs also approximates their answer set semantics. That is, every well-founded (resp., unfounded) atom $a \in H B_{P}$ is true (resp., false) in every answer set. 


\section{Probabilistic Description Logic Programs}

In this section, we define probabilistic dl-programs (or pdl-programs) as a combination of dl-programs with Poole's independent choice logic (ICL) [35]. Poole's ICL is based on ordinary acyclic logic programs under different "choices", where every choice along with an acyclic logic program produces a first-order model, and one then obtains a probability distribution over the set of all first-order models by placing a probability distribution over the different choices. Here, we use stratified dl-programs under their canonical semantics, as well as dl-programs under the well-founded and the answer set semantics, instead of the above ordinary acyclic logic programs under their canonical semantics (which coincides with their stable model semantics and their answer set semantics, respectively).

\subsection{Syntax}

We now define the syntax of pdl-programs and probabilistic queries addressed to them. We first define probabilistic formulas and probabilities on choice spaces.

We assume a function-free first-order vocabulary $\Phi$ with nonempty finite sets of constant and predicate symbols, and a set of variables $\mathcal{X}$, as in Section 3.1. We use $H B_{\Phi}$ (resp., $H U_{\Phi}$ ) to denote the Herbrand base (resp., universe) over $\Phi$. In the sequel, we assume that $H B_{\Phi}$ is nonempty. We define classical formulas by induction as follows. The propositional constants false and true, denoted $\perp$ and $T$, respectively, and all atoms are classical formulas. If $\phi$ and $\psi$ are classical formulas, then $\neg \phi$ and $(\phi \wedge \psi)$ are also classical formulas. A conditional constraint is of the form $(\psi \mid \phi)[l, u]$ with reals $l, u \in[0,1]$ and classical formulas $\phi$ and $\psi$. We define probabilistic formulas inductively as follows. Every conditional constraint is a probabilistic formula. If $F$ and $G$ are probabilistic formulas, then also $\neg F$ and $(F \wedge G)$. We use $(F \vee G),(F \Leftarrow G)$, and $(F \Leftrightarrow G)$ to abbreviate $\neg(\neg F \wedge \neg G), \neg(\neg F \wedge G)$, and $(\neg(\neg F \wedge G) \wedge \neg(F \wedge \neg G))$, respectively, and adopt the usual conventions to eliminate parentheses. Ground terms, ground formulas, substitutions, and ground instances of probabilistic formulas are defined as usual.

A choice space $C$ is a set of pairwise disjoint and nonempty sets $A \subseteq H B_{\Phi}$. Any member $A \in C$ is an alternative of $C$ and any element $a \in A$ an atomic choice of $C$. A total choice of $C$ is a set $B \subseteq H B_{\Phi}$ such that $|B \cap A|=1$ for all $A \in C$. A probability $\mu$ on a choice space $C$ is a probability function on the set of all total choices of $C$. Since $C$ and all its alternatives are finite, $\mu$ can be defined by (i) a mapping $\mu: \bigcup C \rightarrow[0,1]$ such that $\sum_{a \in A} \mu(a)=1$ for all $A \in C$, and (ii) $\mu(B)=\Pi_{b \in B} \mu(b)$ for all total choices $B$ of $C$. Intuitively, (i) associates a probability with each atomic choice of $C$, and (ii) assumes independence between the alternatives of $C$.

A probabilistic dl-program (or pdl-program) $K B=(L, P, C, \mu)$ consists of a dl-program $(L, P)$, a choice space $C$ such that no atomic choice in $C$ coincides with the head of any dl-rule in $\operatorname{ground}(P)$ (note that this condition ensures that stratified pdl-programs are always consistent; cf. Section 4.2), and a probability $\mu$ on $C$. Intuitively, since the total choices of $C$ select subsets of $P$, every probabilistic dl-program is the compact representation of a probability distribution on a finite set of dl-programs. A probabilistic query to $K B$ has the form ?F or the form ? $(\beta \mid \alpha)[R, S]$, where $F$ is a probabilistic formula, $\beta, \alpha$ are classical formulas, and $R, S$ are variables. The correct answer to ?F is the set of all substitutions $\theta$ such that $F \theta$ is a consequence of $K B$. The tight answer to ? $(\beta \mid \alpha)[R, S]$ is the set of all substitutions $\theta$ such that $?(\beta \mid \alpha)[R, S] \theta$ is a tight consequence of $K B$. In the following paragraphs, we define the notions of consequence and tight consequence under the stratified, the answer set, and the well-founded semantics.

Example 4.1 Consider the pdl-program $K B_{1}=\left(L_{1}, P_{1}, C_{1}, \mu_{1}\right)$, where $L_{1}$ and $P_{1}$ are as in Example 2.1 resp. 3.1 except that the dl-rules (4) and (5) are replaced by the dl-rules $\left(4^{\prime}\right)$ and $\left(5^{\prime}\right)$, respectively, and the 
dl-rules (10) and (11) are added:

$\left(4^{\prime}\right)$ avoid $(X) \leftarrow D L[C a m e r a](X)$, not offer $(X)$, avoid_pos;

$\left(5^{\prime}\right) \quad$ offer $(X) \leftarrow D L[P C \uplus p c ;$ Electronics $](X)$, not brand_new $(X)$, offer_pos;

(10) buy $(C, X) \leftarrow$ needs $(C, X)$, view $(X)$, notavoid $(X), v_{-}$buy_pos;

(11) buy $(C, X) \leftarrow$ needs $(C, X)$, buy $(C, Y)$, also_buy $(Y, X)$, a_buy_pos.

Let $C_{1}=\left\{\{\right.$ avoid_pos, avoid_neg $\},\{$ offer_pos, offer_neg $\},\left\{v_{-} b u y_{-} p o s, v_{-} b u y_{-} n e g\right\},\left\{a_{-} b u y_{-} p o s\right.$, $\left.\left.a_{-} b u y_{\_} n e g\right\}\right\}$, and let $\mu_{1}$ be given by $\mu_{1}$ (avoid_pos $)=0.9, \mu_{1}$ (avoid_neg $)=0.1, \mu_{1}$ (offer_pos $)=$ $0.9, \mu_{1}($ offer_neg $)=0.1, \mu_{1}\left(v_{-}\right.$buy_pos $)=0.7, \mu_{1}\left(v_{-} b u y_{-} n e g\right)=0.3, \mu_{1}\left(a_{-} b u y_{-} p o s\right)=0.7$, and $\mu_{1}\left(a_{-}\right.$buy_neg $)=0.3$.

Here, the new dl-rules $\left(4^{\prime}\right)$ and $\left(5^{\prime}\right)$ express that the dl-rules (4) and (5) actually only hold with the probability 0.9 . Furthermore, (10) expresses that a customer buys a needed product that is viewed and not avoided with the probability 0.7 , while (11) says that a customer buys a needed product $x$ with probability 0.7 , if she bought another product $y$, and every customer that previously had bought $y$ also bought $x$.

In a probabilistic query, one may ask for the tight probability bounds that a customer $c$ buys a needed product $x$, if (i) $c$ bought another product $y$, (ii) every customer that previously had bought $y$ also bought $x$, (iii) $x$ is not avoided, and (iv) $c$ has been shown product $x$ (the result to this query may, e.g., help to decide whether it is useful to make a customer automatically also view product $x$ when buying $y$ ):

$$
?(\operatorname{buy}(c, x) \mid \operatorname{needs}(c, x) \wedge \text { buy }(c, y) \wedge \text { also_buy }(y, x) \wedge \text { view }(x) \wedge \neg \text { avoid }(x))[R, S] \text {. }
$$

\subsection{Semantics of Stratified PDL-Programs}

A stratified pdl-program is a pdl-program $K B=(L, P, C, \mu)$ such that the dl-program $(L, P)$ is stratified. In the following, we define the semantic notions of consequence and tight consequence for stratified pdlprograms.

Example 4.2 Consider again the pdl-program $K B_{1}=\left(L_{1}, P_{1}, C_{1}, \mu_{1}\right)$ given in Example 4.1. It is not difficult to see that $K B_{1}$ is stratified.

A total world $I$ is a subset of $H B_{\Phi}$. We use $\mathcal{I}_{\Phi}$ to denote the set of all total worlds over $\Phi$. A variable assignment $\sigma$ maps each variable $X \in \mathcal{X}$ to an element of $H U_{\Phi}$. We extend $\sigma$ to all terms by $\sigma(c)=c$ for all constant symbols $c$ from $\Phi$. The truth of classical formulas $\phi$ in $I$ under a variable assignment $\sigma$, denoted $I \models_{\sigma} \phi$ (or $I \models \phi$ when $\phi$ is ground), is inductively defined by:

- $I \models_{\sigma} p\left(t_{1}, \ldots, t_{k}\right)$ iff $p\left(\sigma\left(t_{1}\right), \ldots, \sigma\left(t_{k}\right)\right) \in I$;

- $I \models_{\sigma} \neg \phi$ iff not $I \models_{\sigma} \phi$; and $I \models_{\sigma}(\phi \wedge \psi)$ iff $I \models_{\sigma} \phi$ and $I \models_{\sigma} \psi$.

A total probabilistic interpretation $\operatorname{Pr}$ is a probability function on $\mathcal{I}_{\Phi}$ (that is, since $\mathcal{I}_{\Phi}$ is finite, a mapping $\operatorname{Pr}: \mathcal{I}_{\Phi} \rightarrow[0,1]$ such that all $\operatorname{Pr}(I)$ with $I \in \mathcal{I}_{\Phi}$ sum up to 1$)$. The probability of a classical formula $\phi$ in $\operatorname{Pr}$ under a variable assignment $\sigma$, denoted $\operatorname{Pr}_{\sigma}(\phi)$ (or $\operatorname{Pr}(\phi)$ when $\phi$ is ground), is defined as the sum of all $\operatorname{Pr}(I)$ such that $I \in \mathcal{I}_{\Phi}$ and $I \models_{\sigma} \phi$. For classical formulas $\phi$ and $\psi$ with $\operatorname{Pr}_{\sigma}(\phi)>0$, we use $\operatorname{Pr}_{\sigma}(\psi \mid \phi)$ to abbreviate $\operatorname{Pr}_{\sigma}(\psi \wedge \phi) / \operatorname{Pr}_{\sigma}(\phi)$. The truth of probabilistic formulas $F$ in $\operatorname{Pr}$ under $\sigma$, denoted $\operatorname{Pr} \models_{\sigma} F$, is inductively defined as follows:

- $\operatorname{Pr} \models_{\sigma}(\psi \mid \phi)[l, u]$ iff $\operatorname{Pr}_{\sigma}(\phi)=0$ or $\operatorname{Pr}_{\sigma}(\psi \mid \phi) \in[l, u]$; 
- $\operatorname{Pr} \models_{\sigma} \neg F$ iff not $\operatorname{Pr} \models_{\sigma} F$; and $\operatorname{Pr} \models_{\sigma}(F \wedge G)$ iff $\operatorname{Pr} \models_{\sigma} F$ and $\operatorname{Pr} \models_{\sigma} G$.

A total probabilistic interpretation $\operatorname{Pr}$ is a model of a probabilistic formula $F$ iff $\operatorname{Pr} \models_{\sigma} F$ for every variable assignment $\sigma$. We say that $\operatorname{Pr}$ is the canonical model of a stratified pdl-program $K B=(L, P, C, \mu)$ iff every world $I \in \mathcal{I}_{\Phi}$ with $\operatorname{Pr}(I)>0$ is the canonical model of $(L, P \cup\{p \leftarrow \mid p \in B\})$ for some total choice $B$ of $C$ such that $\operatorname{Pr}(I)=\mu(B)$. Observe that every stratified pdl-program $K B$ has a unique canonical model $\operatorname{Pr}$. A probabilistic formula $F$ is a consequence of $K B$, denoted $K B \| \sim F$, iff the canonical model of $K B$ is also a model of $F$. A conditional constraint $(\psi \mid \phi)[l, u]$ is a tight consequence of $K B$, denoted $K B \|{ }_{\text {tight }}(\psi \mid \phi)[l, u]$, iff $l$ (resp., $u$ ) is the infimum (resp., supremum) of $\operatorname{Pr}_{\sigma}(\psi \mid \phi)$ subject to the canonical model $\operatorname{Pr}$ of $K B$ and all variable assignments $\sigma$ with $\operatorname{Pr}_{\sigma}(\phi)>0$. Note that query processing in stratified pdl-programs is discussed in Section 5 below.

Example 4.3 Consider again the pdl-program $K B_{1}=\left(L_{1}, P_{1}, C_{1}, \mu_{1}\right)$ given in Example 4.1. Since $\left(L_{1}, P_{1}\right)$ is stratified, also $K B_{1}$ is stratified. The choice space $C_{1}$ has 16 total choices, and each of these total choices is associated with a probability under $\mu_{1}$. For example, one total choice of $C_{1}$ is given by $B_{1}=\{$ avoid_pos, offer_pos, v_buy_pos, a_buy_pos $\}$; it has the probability $\mu_{1}\left(B_{1}\right)=0.9 \times 0.9 \times 0.7 \times 0.7=0.3969$. Every total choice $B$ of $C_{1}$ specifies a canonical model of a stratified dl-program, namely, the canonical model of $(L, P \cup\{p \leftarrow \mid p \in B\})$, which is associated with the probability $\mu_{1}(B)$. Hence, the canonical model $\operatorname{Pr}_{1}$ of $K B_{1}$ consists of 16 canonical models of stratified dl-programs along with their probabilities. For example, the canonical model $I_{B_{1}}$ for the above total choice $B_{1}$ satisfies (among others) the ground atoms in lines (1) to (3) of Example 3.1 as well as offer $(p c 3)$, offer $\left(p c_{-} i b m\right)$, and offer $\left(p c_{-} h p\right)$; the canonical model $I_{B_{1}}$ has the associated probability $\mu_{1}\left(B_{1}\right)=0.3969$. The canonical model $P r_{1}$ of $K B_{1}$ thus represents exactly one probability distribution over first-order models, and it allows to assign a probability to any ground classical formula. For example, the ground atoms offer $(p c 3)$, offer $\left(p c_{-} i b m\right)$, and offer $\left(p c_{-} h p\right)$ have each the probability 0.9 under the canonical model $P r_{1}$ of $K B_{1}$ (beside $I_{B_{1}}$ there are other $I_{B}$ that satisfy offer $(p c 3)$, offer $\left(p c_{-} i b m\right)$, and offer $\left.\left(p c_{-} h p\right)\right)$.

\subsection{Answer Set Semantics of PDL-Programs}

We now introduce the notions of consistency, consequence, and tight consequence under the answer set semantics for general pdl-programs.

A total probabilistic interpretation $P r$ is an answer set model of a pdl-program $K B=(L, P, C, \mu)$ iff (i) every total world $I \in \mathcal{I}_{\Phi}$ with $\operatorname{Pr}(I)>0$ is an answer set of $(L, P \cup\{p \leftarrow \mid p \in B\})$ for some total choice $B$ of $C$, and (ii) $\operatorname{Pr}(\bigwedge B)=\operatorname{Pr}\left(\bigwedge_{p \in B} p\right)=\mu(B)$ for every total choice $B$ of $C$. We say that $K B$ is consistent iff it has an answer set model $\mathrm{Pr}$. A probabilistic formula $F$ is an answer set consequence of $K B$, denoted $K B \|{ }^{a s} F$, iff every answer set model of $K B$ is also a model of $F$. A conditional constraint $(\psi \mid \phi)[l, u]$ is a tight answer set consequence of $K B$, denoted $K B \|{ }_{t i g h t}^{a s}(\psi \mid \phi)[l, u]$, iff $l$ (resp., $u$ ) is the infimum (resp., supremum) of $\operatorname{Pr}_{\sigma}(\psi \mid \phi)$ subject to all answer set models $\operatorname{Pr}$ of $K B$ and all variable assignments $\sigma$ with $\operatorname{Pr}_{\sigma}(\phi)>0$. Here, we assume that $l=1$ and $u=0$, when $\operatorname{Pr}_{\sigma}(\phi)=0$ for all answer set models $\operatorname{Pr}$ of $K B$ and all $\sigma$.

Every stratified pdl-program $K B$ is consistent and has exactly one answer set model, which coincides with the canonical model of $K B$. Deciding whether a general pdl-program $K B$ is consistent can be reduced to deciding whether dl-programs have an answer set. The following theorem shows that computing tight answers to queries ? $(\beta \mid \alpha)[R, S]$ to $K B$, where $\beta$ and $\alpha$ are ground, can be reduced to computing all answer sets of dl-programs and then solving two linear optimization problems. It follows from a standard result on transforming linear fractional programs into equivalent linear programs by Charnes and Cooper [7]. 


$$
\begin{array}{rlrl}
\sum_{r \in R, r \nvdash \wedge B}-\mu(B) y_{r}+\sum_{r \in R, r \models \wedge B}(1-\mu(B)) y_{r} & =0 & & (\text { for all total choices } B \text { of } C) \\
\sum_{r \in R, r \models \alpha} y_{r} & =1 & \\
y_{r} & \geq 0 & & (\text { for all } r \in R)
\end{array}
$$

Figure 1: System of linear constraints $L C$ for Theorem 4.4.

Theorem 4.4 Let $K B=(L, P, C, \mu)$ be a consistent pdl-program, and let $\beta$ and $\alpha$ be ground classical formulas such that $\operatorname{Pr}(\alpha)>0$ for some answer set model $\operatorname{Pr}$ of $K B$. Then, l (resp., u) such that $K B \|{ }_{\text {tight }}^{\text {as }}$ $(\beta \mid \alpha)[l, u]$ is the optimal value of the following linear program over the variables $y_{r}(r \in R)$, where $R$ is the union of all sets of answer sets of $(L, P \cup\{p \leftarrow \mid p \in B\})$ for all total choices $B$ of $C$ :

minimize (resp., maximize) $\sum_{r \in R, r \models \beta \wedge \alpha} y_{r} \quad$ subject to LC in Fig. 1.

\subsection{Well-Founded Semantics of PDL-Programs}

We finally define the notions of consequence and tight consequence under the well-founded semantics for general pdl-programs. We first define partial probabilistic interpretations and the evaluation of probabilistic formulas in them.

A partial world $I$ is a consistent subset of $L i t_{\Phi}=H B_{\Phi} \cup \neg . H B_{\Phi}$. We identify $I$ with the three-valued interpretation $I: H B_{\Phi} \rightarrow$ true, false, undefined $\}$ that is defined by $I(a)=$ true iff $a \in I, I(a)=$ false iff $\neg a \in I$, and $I(a)=$ undefined iff $I \cap\{a, \neg a\}=\emptyset)$. We use $\mathcal{I}_{\Phi}^{p}$ to denote the set of all partial worlds over $\Phi$. Every classical formula $\phi$ in a partial world $I$ under a variable assignment $\sigma$ is associated with a threevalued truth value from \{true, false, undefined\}, denoted $I_{\sigma}(\phi)$ (or simply $I(\phi)$ when $\phi$ is ground), which is inductively defined by:

- $I_{\sigma}\left(p\left(t_{1}, \ldots, t_{k}\right)\right)=I\left(p\left(\sigma\left(t_{1}\right), \ldots, \sigma\left(t_{k}\right)\right)\right)$;

- $I_{\sigma}(\neg \phi)=$ true iff $I_{\sigma}(\phi)=$ false, and $I_{\sigma}(\neg \phi)=$ false iff $I_{\sigma}(\phi)=$ true;

- $I_{\sigma}(\phi \wedge \psi)=$ true iff $I_{\sigma}(\phi)=I_{\sigma}(\psi)=$ true, and $I_{\sigma}(\phi \wedge \psi)=$ false iff $I_{\sigma}(\phi)=$ false or $I_{\sigma}(\psi)=$ false.

A partial probabilistic interpretation $\operatorname{Pr}$ is a probability function on $\mathcal{I}_{\Phi}^{p}$. The probability of a classical formula $\phi$ in $\operatorname{Pr}$ under a variable assignment $\sigma$, denoted $\operatorname{Pr}_{\sigma}(\phi)$ (or simply $\operatorname{Pr}(\phi)$ when $\phi$ is ground), is undefined, if $I_{\sigma}(\phi)$ is undefined for some $I \in \mathcal{I}_{\Phi}^{p}$ with $\operatorname{Pr}(I)>0$; and $\operatorname{Pr}_{\sigma}(\phi)$ is defined as the sum of all $\operatorname{Pr}(I)$ such that $I \in \mathcal{I}_{\Phi}^{p}$ and $I_{\sigma}(\phi)=$ true, otherwise. For classical formulas $\phi$ and $\psi$ such that $\operatorname{Pr}_{\sigma}(\phi)>0$, the conditional probability of $\psi$ given $\phi$ in $\operatorname{Pr}$ under $\sigma$, denoted $\operatorname{Pr}_{\sigma}(\psi \mid \phi)$, is defined as $\operatorname{Pr}_{\sigma}(\psi \wedge \phi) / \operatorname{Pr}_{\sigma}(\phi)$. Note that, alternatively, we may also define $\operatorname{Pr}_{\sigma}(\phi)$ as the interval $\left[\sum_{I_{\sigma}(\phi)=\text { true }}\right.$ $\left.\operatorname{Pr}(I), 1-\sum_{I_{\sigma}(\phi)=\text { false }} \operatorname{Pr}(I)\right]$. However, even though this definition ensures that $\operatorname{Pr}_{\sigma}(\phi)$ is always defined, it cannot easily be generalized to conditional probabilities. Every probabilistic formula $F$ in $\operatorname{Pr}$ under $\sigma$ is associated with a three-valued truth value from \{true, false, undefined\}, denoted $\operatorname{Pr}_{\sigma}(F)$, which is inductively defined as follows: 
- $\operatorname{Pr}_{\sigma}((\psi \mid \phi)[l, u])=$ true iff $\operatorname{Pr}_{\sigma}(\phi)=0$ or $\operatorname{Pr}_{\sigma}(\psi \mid \phi) \in[l, u]$, and $\operatorname{Pr}_{\sigma}((\psi \mid \phi)[l, u])=$ false iff $\operatorname{Pr}_{\sigma}(\phi)>0$ and $\operatorname{Pr}_{\sigma}(\psi \mid \phi) \notin[l, u]$;

- $\operatorname{Pr}_{\sigma}(\neg F)=$ true iff $\operatorname{Pr}_{\sigma}(F)=$ false, and $\operatorname{Pr}_{\sigma}(\neg F)=$ false iff $\operatorname{Pr}_{\sigma}(F)=$ true;

- $\operatorname{Pr}_{\sigma}(F \wedge G)=$ true iff $\operatorname{Pr}_{\sigma}(F)=\operatorname{Pr}_{\sigma}(G)=$ true, and $\operatorname{Pr}_{\sigma}(F \wedge G)=$ false iff $\operatorname{Pr}_{\sigma}(F)=$ false or $\operatorname{Pr}_{\sigma}(G)=$ false.

The well-founded model of a pdl-program $K B=(L, P, C, \mu)$, denoted $\operatorname{Pr}_{K B}^{w f}$, is defined as follows: (i) $\operatorname{Pr}_{K B}^{w f}\left(I_{B}\right)=\mu(B)$, where $I_{B}$ is the well-founded model of $(L, P \cup\{p \leftarrow \mid p \in B\})$, for every total choice $B$ of $C$, and (ii) $\operatorname{Pr}_{K B}^{w f}(I)=0$ for all other $I \in \mathcal{I}_{\Phi}^{p}$. A probabilistic formula $F$ is a well-founded consequence of $K B$, denoted $K B \| \sim^{w f} F$, iff $F$ is true in $\operatorname{Pr}_{K B}^{w f}$ under every variable assignment $\sigma$. A conditional constraint $(\psi \mid \phi)[l, u]$ is a tight well-founded consequence of $K B$, denoted $K B \|_{\text {tight }}^{w f}(\psi \mid \phi)[l, u]$, iff (i) $\operatorname{Pr}_{K B}^{w f}(\phi)$ under every variable assignments $\sigma$ is different from undefined, and (ii) $l$ (resp., $u$ ) is the infimum (resp., supremum) of $\operatorname{Pr}_{\sigma}(\psi \mid \phi)$ subject to $\operatorname{Pr}=\operatorname{Pr}_{K B}^{w f}$ and all variable assignments $\sigma$ with $\operatorname{Pr}_{\sigma}(\phi)>0$.

The well-founded model of a stratified pdl-program $K B$ is total and coincides with the canonical model of $K B$. As an advantage of the well-founded semantics, every general pdl-program $K B$ has a unique wellfounded model, but not necessarily an answer set model. Furthermore, the unique well-founded model can be easily computed by fixpoint iteration [13]. As a drawback, the well-founded model associates only with some classical formulas under $\sigma$ a probability, while every answer set model associates with all classical formulas under $\sigma$ a probability. The following theorem shows that the answer set semantics is a refinement of the well-founded semantics. That is, if an answer to a query under the well-founded semantics is defined, then it coincides with the answer under the answer set semantics. The theorem follows from the result that the well-founded semantics of dl-programs approximates their answer set semantics [13]. The advantages of both semantics can thus be combined in query processing by first trying to compute the well-founded answer, and only if this does not exist the answer under the answer set semantics.

Theorem 4.5 Let $K B=(L, P, C, \mu)$ be a consistent pdl-program, and let $(\psi \mid \phi)[l, u]$ be a ground conditional constraint. If $\operatorname{Pr}_{K B}^{w f}(\phi), \operatorname{Pr}_{K B}^{w f}(\psi \wedge \phi) \neq$ undefined, then

(a) $K B \| \sim^{w f}(\psi \mid \phi)[l, u]$ iff $K B \mathbb{\sim}^{\text {as }}(\psi \mid \phi)[l, u]$, and

(b) $K B \| \sim_{\text {tight }}^{w f}(\psi \mid \phi)[l, u]$ iff $K B \mathbb{\sim}_{\text {tight }}^{\text {as }}(\psi \mid \phi)[l, u]$.

Proof (sketch). Let $\alpha \in\{\psi, \psi \wedge \phi\}$. It is sufficient to show that $\operatorname{Pr}_{K B}^{w f}(\alpha)$ is equal to $\operatorname{Pr}(\alpha)$ for all answer set models $\operatorname{Pr}$ of $K B$. Observe that $\operatorname{Pr}_{K B}^{w f}(\alpha)$ is the sum of all $\mu(B)$ such that (i) $B$ is a total choice of $C$, (ii) $\mu(B)>0$, and (iii) $I_{B}(\alpha)=$ true, where $I_{B}$ denotes the well-founded model of $(L, P \cup\{p \leftarrow \mid p \in B\})$. By induction on the structure of classical formulas, it is not difficult to see that $I_{B}(\alpha)=$ true iff $I \models \alpha$ for all answer sets $I$ of $(L, P \cup\{p \leftarrow \mid p \in B\})$. This already shows that $\operatorname{Pr}_{K B}^{w f}(\alpha)$ is equal to $\operatorname{Pr}(\alpha)$ for all answer set models $\operatorname{Pr}$ of $K B$.

\section{Query Processing in Stratified PDL-Programs}

The canonical model of an ordinary positive (resp., stratified) normal program $P$ has a fixpoint characterization in terms of an immediate consequence operator $T_{P}$, which generalizes to positive (resp., stratified) 
dl-programs. This can be used for a bottom-up computation of the canonical model of a positive (resp., stratified) dl-program, and thus also for computing the canonical model of a stratified pdl-program and for query processing in stratified pdl-programs.

\subsection{Fixpoint Iteration in Positive DL-Programs}

We first describe a fixpoint characterization of the canonical model of a positive dl-program. For any dl-program $K B=(L, P)$, we define the operator $T_{K B}$ on the subsets of $H B_{P}$ as follows. For every $I \subseteq H B_{P}$, let

$$
T_{K B}(I)=\left\{H(r) \mid r \in \operatorname{ground}(P), I=_{L} \ell \text { for all } \ell \in B(r)\right\} .
$$

If $K B$ is positive, then $T_{K B}$ is monotonic. Hence, $T_{K B}$ has a least fixpoint, denoted $l f p\left(T_{K B}\right)$. Furthermore, lfp $\left(T_{K B}\right)$ can be computed by a finite fixpoint iteration (given finiteness of $P$ and the number of constant symbols in $\Phi)$. For every $I \subseteq H B_{P}$, we define $T_{K B}^{i}(I)=I$, if $i=0$, and $T_{K B}^{i}(I)=T_{K B}\left(T_{K B}^{i-1}(I)\right)$, if $i>0$.

Theorem 5.1 For every positive dl-program $K B=(L, P)$, it holds that lfp $\left(T_{K B}\right)=M_{K B}$. Furthermore, lfp $\left(T_{K B}\right)=\bigcup_{i=0}^{n} T_{K B}^{i}(\emptyset)=T_{K B}^{n}(\emptyset)$ for some $n \geq 0$.

\subsection{Fixpoint Iteration in Stratified DL-Programs}

We next describe a fixpoint characterization for stratified dl-programs. Using Theorem 5.1, we can characterize the canonical model $M_{K B}$ of a stratified dl-program $K B=(L, P)$ as follows. Let $\widehat{T}_{K B}^{i}(I)=T_{K B}^{i}(I) \cup I$ for all $i \geq 0$.

Theorem 5.2 Suppose that $K B=(L, P)$ has a stratification $\lambda$ of length $k \geq 0$. Let $M_{i} \subseteq H B_{P}, i \in\{-1,0$, $\ldots, k\}$, be defined by $M_{-1}=\emptyset$ and $M_{i}=\widehat{T}_{K B_{i}}^{n_{i}}\left(M_{i-1}\right)$ for $i \geq 0$, where $n_{i} \geq 0$ such that $\widehat{T}_{K B_{i}}^{n_{i}}\left(M_{i-1}\right)=$ $\widehat{T}_{K B_{i}}^{n_{i}+1}\left(M_{i-1}\right)$. Then, $M_{k}=M_{K B}$.

\subsection{Query Processing in Stratified PDL-Programs}

Algorithm canonical_model (see Fig. 2) computes the canonical model $\operatorname{Pr}$ of a given stratified pdl-program $K B=(L, P, C, \mu)$. It is essentially based on a reduction to computing the canonical model of stratified dl-programs in line 4 , which can be done using the above finite sequence of finite fixpoint iterations.

Example 5.3 Consider again the stratified pdl-program $K B_{1}=\left(L_{1}, P_{1}, C_{1}, \mu_{1}\right)$ of Example 4.1. The computation of canonical_model in Fig. 2 on $K B_{1}$ is summarized as follows. For each of the 16 total choices $B$ of $C_{1}$, the canonical model $I_{B}$ of the stratified dl-program $\left(L_{1}, P_{1} \cup\{p \leftarrow \mid p \in B\}\right)$ is computed, and $I_{B}$ is associated with the probability $\mu_{1}(B)$, while all the other $I \in \mathcal{I}_{\Phi}$ are associated with the probability 0 . For example, for the total choice $B_{1}=\{$ avoid_pos, offer_pos, v_buy_pos, a_buy_pos $\}$ of $C_{1}$, the canonical model $I_{B_{1}}$ of the stratified dl-program $\left(L_{1}, P_{1} \cup\left\{p \leftarrow \mid p \in B_{1}\right\}\right)$ satisfies exactly the ground atoms in lines (1) to (3) of Example 3.1 as well as offer (pc3), offer $\left(p c_{\_} i b m\right)$, offer $\left(p c_{\_} h p\right)$, provider(dell), provider $(i b m)$, provider $(h p)$, similar $\left(t b_{-} a i, t b_{-} l p\right)$, and $\operatorname{similar}\left(p c_{-} i b m, p c_{-} h p\right)$, and $I_{B_{1}}$ is associated with the probability $\mu_{1}\left(B_{1}\right)=0.9 \times 0.9 \times 0.7 \times 0.7=0.3969$. 


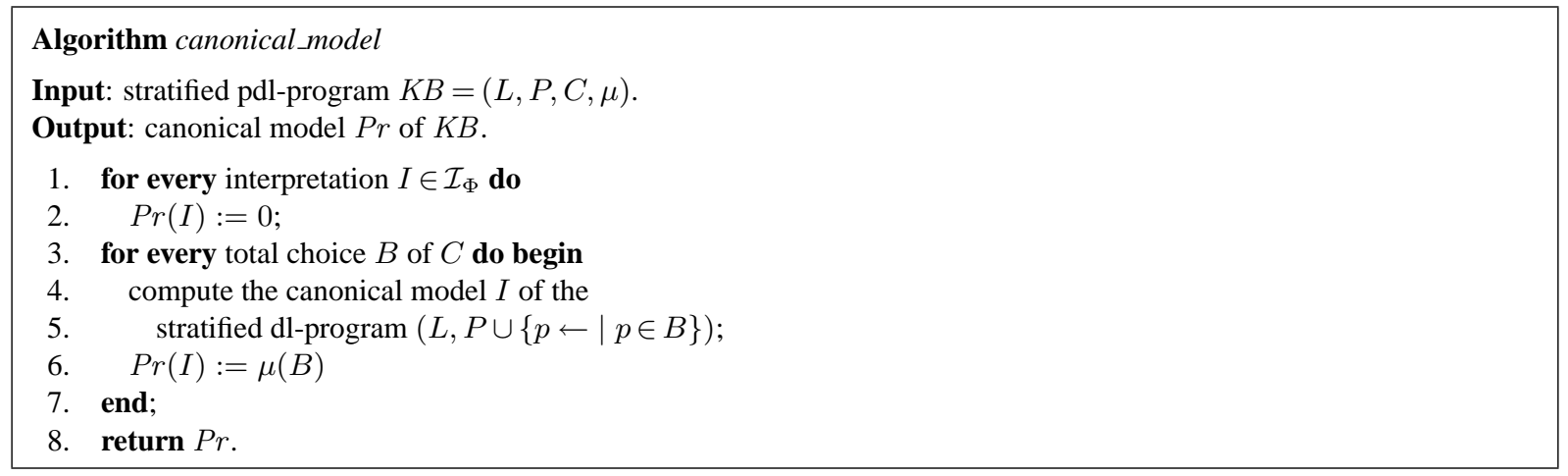

Figure 2: Algorithm canonical_model.

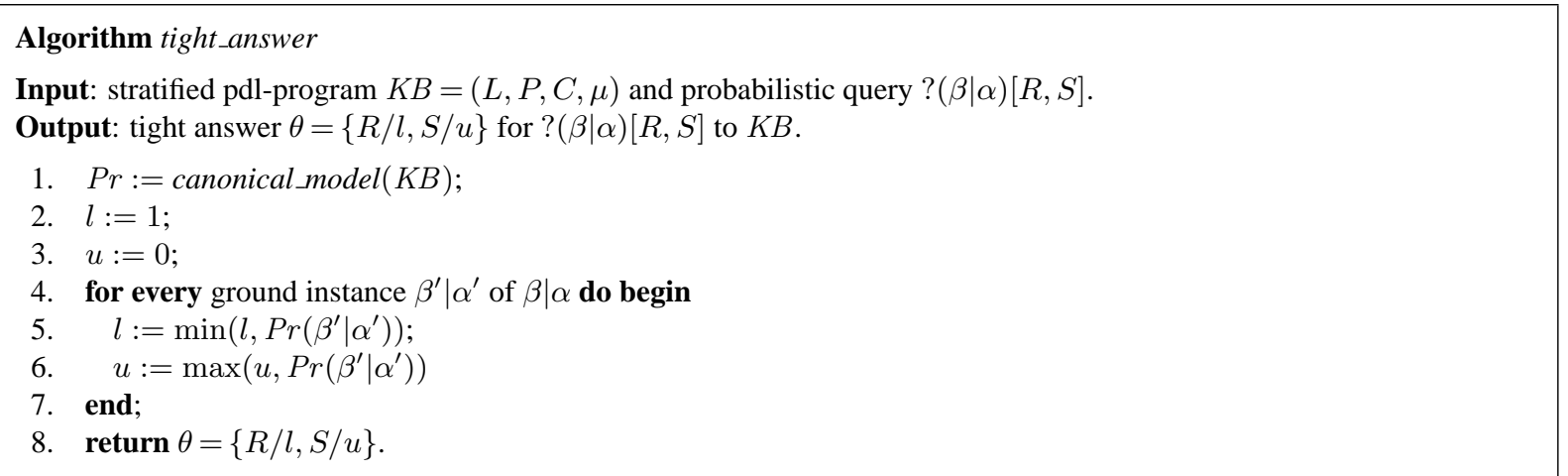

Figure 3: Algorithm tight_answer.

Algorithm tight_answer (see Fig. 3) computes tight answers $\theta=\{R / l, S / u\}$ for a given probabilistic query $?(\beta \mid \alpha)[R, S]$ to a given stratified pdl-program $K B$. It computes the canonical model of $K B$ in line 1 and the tight answer in lines $2-8$.

Example 5.4 To compute the tight answer for the query?(offer $(p c 3) \mid \top)[R, S]$ to the stratified pdl-program $K B_{1}=\left(L_{1}, P_{1}, C_{1}, \mu_{1}\right)$ of Example 4.1, Algorithm tight_answer in Fig. 3 first computes the canonical model $\operatorname{Pr}$ of $K B_{1}$, using Algorithm canonical_model. Then, since $\beta|\alpha=\operatorname{offer}(p c 3)| \top$ is ground and unconditional, Algorithm tight_answer computes only the probability of offer (pc3) in $\operatorname{Pr}$. Finally, since the latter is given by 0.9 , the algorithm returns $\theta=\{R / 0.9, S / 0.9\}$.

\section{Related Work}

In this section, we discuss related work on the combination of logic programs with description logics and on uncertainty reasoning for the Semantic Web. Note that an overview of the large body of previous work on the combination of logic programs with probabilistic uncertainty is contained in $[30,26]$. 


\subsection{Description Logic Programs}

Related work on the combination of description logics and logic programs can be divided into (a) hybrid approaches using description logics as input to logic programs, (b) approaches reducing description logics to logic programs, (c) combinations of description logics with default and defeasible logic, and (d) approaches to rule-based well-founded reasoning in the Semantic Web. Below we give some representatives for (a)-(d). Further works and details are given in $[12,13]$.

The works by Donini et al. [10], Levy and Rousset [27], and Rosati [37, 38] are representatives of hybrid approaches using description logics as input. Donini et al. [10] introduce a combination of (disjunction, negation-, and function-free) datalog with the description logic $\mathcal{A L C}$. An integrated knowledge base consists of a structural component in $\mathcal{A L C}$ and a relational component in datalog, where the integration of both components lies in using concepts from the structural component as constraints in rule bodies of the relational component. The closely related work by Levy and Rousset [27] presents a combination of Horn

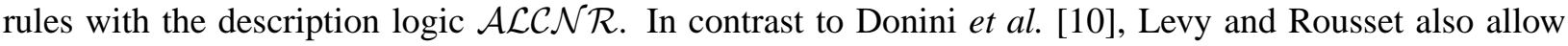
for roles as constraints in rule bodies, and do not require the safety condition that variables in constraints in the body of a rule $r$ must also appear in ordinary atoms in the body of $r$. Finally, Rosati [37] presents a combination of disjunctive datalog (with classical and default negation, but without function symbols) with $\mathcal{A L C}$, which is based on a generalized answer set semantics.

Some approaches reducing description logic reasoning to logic programming are the works by Van Belleghem et al. [44], Alsaç and Baral [1], Swift [43], Grosof et al. [19], and Hufstadt et al. [25]. Early work on dealing with default information in description logics is the approach due to Baader and Hollunder [4], where Reiter's default logic is adapted to terminological knowledge bases. Antoniou [2] combines defeasible reasoning with description logics for the Semantic Web. In [3], Antoniou and Wagner summarize defeasible and strict reasoning in a single rule formalism.

An important approach to rule-based reasoning under the well-founded semantics for the Semantic Web is due to Damásio [9]. He aims at Prolog tools for implementing different semantics for RuleML [6]. So far, an XML parser library as well as a RuleML compiler have been developed, with routines to convert RuleML rule bases to Prolog and vice versa. The compiler supports paraconsistent well-founded semantics with explicit negation; it is planned to be extended to use XSB [36].

\subsection{Uncertainty Reasoning for the Semantic Web}

Related approaches to uncertainty reasoning for the Semantic Web can be roughly divided into (a) description logic programs under non-probabilistic uncertainty, (b) probabilistic generalizations of description logics, and (c) probabilistic generalizations of web ontology languages, such as DAML+OIL and OWL.

As for (a), previous works by Straccia combine (positive) description logic programs with lattice-based uncertainty [41] and with fuzzy vagueness [42]. Whereas, to my knowledge, the present paper is the first one combining (normal) description logic programs with probabilistic uncertainty.

As for (b), Giugno and Lukasiewicz [18] present a probabilistic generalization of the expressive description logic $\mathcal{S H O Q}(\mathbf{D})$ that stands behind DAML+OIL, which is based on lexicographic probabilistic reasoning. In earlier work, Heinsohn [20] and Jaeger [28] present probabilistic extensions to the description logic $\mathcal{A L C}$, which are essentially based on probabilistic reasoning in probabilistic logics. Koller et al. [29] present a probabilistic generalization of the CLASSIC description logic, which uses Bayesian networks as underlying probabilistic reasoning formalism. Note that fuzzy description logics, such as the ones by Straccia $[39,40]$, are less closely related to probabilistic description logics, since they deal with fuzzy vagueness, rather than probabilistic ambiguity and imprecision. 
As for (c), there are especially the works by Costa [8], Pool and Aikin [34], and Ding and Peng [11], which present probabilistic generalizations of the web ontology language OWL. In particular, Costa's work [8] is semantically based on multi-entity Bayesian networks, while [11] has a semantics in standard Bayesian networks. In closely related work, Fukushige [16] proposes a basic framework for representing probabilistic relationships in RDF. Finally, Nottelmann and Fuhr [33] present pDAML+OIL, which is a probabilistic generalization of the web ontology language DAML+OIL, along with a mapping to stratified probabilistic datalog.

\section{Conclusion}

We have presented probabilistic dl-programs (or pdl-programs), which are a combination of dl-programs under the answer set and the well-founded semantics with Poole's independent choice logic. We have shown that query processing in such pdl-programs can be reduced to computing all answer sets of dl-programs and solving linear optimization problems, and to computing the well-founded semantics of dl-programs, respectively. We have also shown that the answer set semantics of pdl-programs is a refinement of the well-founded semantics of pdl-programs. Moreover, we have considered the special case of stratified pdlprograms. In particular, we have presented an algorithm for query processing in such pdl-programs, which is based on a reduction to computing the canonical model of stratified dl-programs.

An interesting topic of future research is to further enhance pdl-programs towards a possible use for Web Services. This may be done by exploiting and generalizing further features of Poole's ICL for dynamic and multi-agent systems [35]. It would also be interesting to further explore the computational aspects of query processing in pdl-programs under the stratified, answer set, and well-founded semantics.

\section{References}

[1] G. Alsaç and C. Baral. Reasoning in description logics using declarative logic programming. Technical Report, Department of Computer Science and Engineering, Arizona State University, 2001.

[2] G. Antoniou. Nonmonotonic rule systems on top of ontology layers. In Proceedings ISWC-2002, LNCS 2342, pp. 394-398, 2002.

[3] G. Antoniou and G. Wagner. Rules and defeasible reasoning on the Semantic Web. In Proceedings RuleML-2003, LNCS 2876, pp. 111-120, 2003.

[4] F. Baader and B. Hollunder. Embedding defaults into terminological representation systems. J. Autom. Reason., 14:149-180, 1995.

[5] T. Berners-Lee. Weaving the Web. Harper, San Francisco, CA, USA, 1999.

[6] H. Boley, S. Tabet, and G. Wagner. Design rationale for RuleML: A markup language for Semantic Web rules. In Proceedings SWWS-2001, pp. 381-401, 2001.

[7] A. Charnes and W. W. Cooper. Programming with linear fractional functionals. Naval Research Logistics Quarterly, 9:181-186, 1962.

[8] P. C. G. da Costa. Bayesian semantics for the Semantic Web. Doctoral Dissertation, George Mason University, Fairfax, VA, USA, 2005. 
[9] C. V. Damásio. The $\mathrm{W}^{4}$ Project, 2002. See http://centria.di.fct.unl.pt/ cd/ projectos/w4/index.htm.

[10] F. M. Donini, M. Lenzerini, D. Nardi, and A. Schaerf. $\mathcal{A} \mathcal{L}$-log: Integrating datalog and description logics. J. Intell. Inf. Syst. (JIIS), 10(3):227-252, 1998.

[11] Z. Ding and Y. Peng. A Probabilistic extension to ontology language OWL. In Proceedings HICSS2004, 2004.

[12] T. Eiter, T. Lukasiewicz, R. Schindlauer, and H. Tompits. Combining answer set programming with description logics for the Semantic Web. In Proceedings KR-2004, pp. 141-151, 2004. Extended Report RR-1843-03-13, Institut für Informationssysteme, TU Wien, 2003.

[13] T. Eiter, T. Lukasiewicz, R. Schindlauer, and H. Tompits. Well-founded semantics for description logic programs in the Semantic Web. In Proceedings RuleML-2004, LNCS 3323, pp. 81-97, 2004.

[14] D. Fensel, W. Wahlster, H. Lieberman, and J. Hendler, editors. Spinning the Semantic Web: Bringing the World Wide Web to Its Full Potential. MIT Press, 2002.

[15] A. Finzi and T. Lukasiewicz. Structure-based causes and explanations in the independent choice logic. In Proceedings UAI-2003, pp. 225-232, 2003.

[16] Y. Fukushige. Representing probabilistic knowledge in the Semantic Web. In Proceedings of the W3C Workshop on Semantic Web for Life Sciences, Cambridge, MA, USA, 2004.

[17] M. Gelfond and V. Lifschitz. Classical negation in logic programs and deductive databases. New Generat. Comput., 17:365-387, 1991.

[18] R. Giugno and T. Lukasiewicz. P-SHOQQ $(\mathbf{D})$ : A probabilistic extension of $\mathcal{S H O} \mathcal{Q}(\mathbf{D})$ for probabilistic ontologies in the Semantic Web. In Proceedings JELIA-2002, LNCS 2424, pp. 86-97, 2002.

[19] B. N. Grosof, I. Horrocks, R. Volz, and S. Decker. Description logic programs: Combining logic programs with description logics. In Proceedings WWW-2003, pp. 48-57, 2003.

[20] J. Heinsohn. Probabilistic description logics. In Proceedings UAI-1994, pp. 311-318, 1994.

[21] I. Horrocks and P. F. Patel-Schneider. Reducing OWL entailment to description logic satisfiability. In Proceedings ISWC-2003, LNCS 2870, pp. 17-29, 2003.

[22] I. Horrocks and P. F. Patel-Schneider. A proposal for an OWL Rules Language. In Proceedings WWW2004, pp. 723-731, 2004.

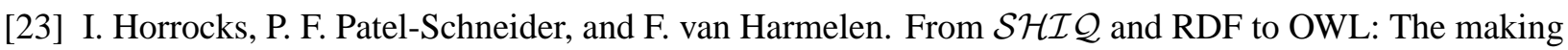
of a web ontology language. J. Web Semantics, 1(1):7-26, 2003.

[24] I. Horrocks, U. Sattler, and S. Tobies. Practical reasoning for expressive description logics. In Proceedings LPAR-1999, LNCS 1705, pp. 161-180, 1999.

[25] U. Hufstadt, B. Motik, and U. Sattler. Reasoning for description logics around $\mathcal{S H \mathcal { I }}$ in a resolution framework. Technical Report 3-8-04/04, FZI Karlsruhe, 2004.

[26] G. Kern-Isberner and T. Lukasiewicz. Combining probabilistic logic programming with the power of maximum entropy. Artif. Intell., 157(1-2):139-202, 2004.

[27] A. Y. Levy and M.-C. Rousset. Combining Horn rules and description logics in CARIN. Artif. Intell., 104(1-2):165-209, 1998. 
[28] M. Jaeger. Probabilistic reasoning in terminological logics. In Proceedings KR-1994, pp. 305-316, 1994.

[29] D. Koller, A. Levy, and A. Pfeffer. P-Classic: A tractable probabilistic description logic. In Proceedings AAAI-1997, pp. 390-397, 1997.

[30] T. Lukasiewicz. Probabilistic logic programming with conditional constraints. ACM Trans. Comput. Log., 2(3):289-339, 2001.

[31] T. Lukasiewicz. Probabilistic description logic programs. In Proceedings ECSQARU-2005, LNCS 3571, pp. 737-749, 2005.

[32] T. Lukasiewicz. Stratified Probabilistic description logic programs. In Proceedings URSW-2005, pp. 87-97, 2005.

[33] H. Nottelmann and N. Fuhr. pDAML+OIL: A probabilistic extension to DAML+OIL based on probabilistic Datalog. In Proceedings IPMU-2004, 2004.

[34] M. Pool and J. Aikin. KEEPER and Protégé: An elicitation environment for Bayesian inference tools. In Proceedings of the Workshop on Protégé and Reasoning held at the 7th International Protégé Conference, 2004.

[35] D. Poole. The independent choice logic for modelling multiple agents under uncertainty. Artif. Intell., 94(1-2):7-56, 1997.

[36] P. Rao, K. Sagonas, T. Swift, D. S. Warren, and J. Freire. XSB: A system for efficiently computing WFS. In Proceedings LPNMR-1997, LNCS 1265, pp. 430-440, 1997.

[37] R. Rosati. Towards expressive KR systems integrating datalog and description logics: Preliminary report. In Proceedings DL-1999, pp. 160-164, 1999.

[38] R. Rosati. On the decidability and complexity of integrating ontologies and rules. J. Web Semantics, 3(1):61-73, 2005.

[39] U. Straccia. Reasoning within fuzzy description logics. J. Artif. Intell. Res., 14:137-166, 2001.

[40] U. Straccia. Towards a fuzzy description logic for the Semantic Web (preliminary report). In Proceedings ESWC-2005, LNCS 3532, pp. 167-181, 2005.

[41] U. Straccia. Uncertainty and description logic programs over lattices. In E. Sanchez, editor, Fuzzy Logic and the Semantic Web, Capturing Intelligence, chapter 7, pp. 115-133. Elsevier, 2006.

[42] U. Straccia. Fuzzy description logic programs. In Proceedings IPMU-2006, 2006.

[43] T. Swift. Deduction in ontologies via ASP. In Proceedings LPNMR-2004, LNCS 2923, pp. 275-288, 2004.

[44] K. Van Belleghem, M. Denecker, and D. De Schreye. A strong correspondence between description logics and open logic programming. In Proceedings ICLP-1997, pp. 346-360, 1997.

[45] A. Van Gelder, K. A. Ross, and J. S. Schlipf. The well-founded semantics for general logic programs. J. ACM, 38(3):620-650, 1991.

[46] W3C. OWL web ontology language overview, 2004. W3C Recommendation (10 Feb. 2004). See www.w3.org/TR/2004/REC-owl-features-20040210/. 